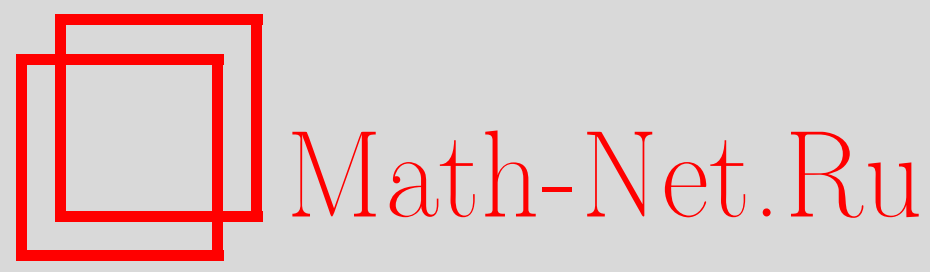

А. Г. Багдасарян, Об интерполяции некоторых обобщенных функциональных пространств разной анизотропии, Матем. заметки, 1997, том 62, выпуск 5, 666-672

DOI: https://doi.org/10.4213/mzm1652

Использование Общероссийского математического портала Math-Net.Ru подразумевает, что вы прочитали и согласны с пользовательским соглашением http://www.mathnet.ru/rus/agreement

Параметры загрузки:

IP : 35.173 .137 .237

26 апреля 2023 г., 10:09:16

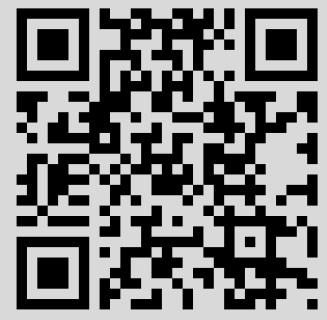




\section{ОБ ИНТЕРПОЛЯЦИИ НЕКОТОРЫХ ОБОБЩЕННЫХ ФУНКЦИОНАЛЬНЫХ ПРОСТРАНСТВ РАЗНОЙ АНИЗОТРОПИИ}

\section{А. Г. Багдасарян}

В работе исследуются интерполяционные свойства пространств $H_{p}^{s}\left(\nu ; \mathbb{R}_{n}\right)$ типа Соболева-Лиувилля и $B_{p, q}^{s}\left(\mu ; \mathbb{R}_{n}\right)$ типа Никольского-Бесова, порожденных бесконечно дифференцируемыми вне нуля функциями полиномиального роста. Доказьваются интерполяционные формулы для пар $\left\{H\left(\nu_{0}\right), H\left(\nu_{1}\right)\right\}$ и $\left\{B\left(\mu_{0}\right), B\left(\mu_{1}\right)\right\}$ пространств указанных типов, причем анизотропии интерполируемьх пространств не зависят друг от друга. Исследуемые пространства при конкретизации порождающих функций совпадают с классическими (изотропными и анизотропными) пространствами Соболева-Лиувилля и Никольского-Бесова.

Библиограффия: 9 названий.

В работе исследуются интерполящионные свойства обобщенных пространств $B_{p, q}^{s}(\mu$; $\left.\mathbb{R}_{n}\right)$ типа Никольского-Бесова и $H_{p}^{s}\left(\nu ; \mathbb{R}_{n}\right)$ типа Соболева-Лиувилля, которые при конкретизации функции $\mu(\xi)$ совпадают с классическими (изотропными и анизотропньми) пространствами Никольского-Бесова и Соболева-Лиувилля (см. [1]).

Изучаемые $B$ - и $H$-пространства естественным образом возникают при исследовании краевых задач для общих регулярных дифференциальных операторов. Однако, при изучении интерполящионньх свойств даже классических анизотропных пространств (с разной анизотропией) приходится рассматривать пространства, порожденные “вполне правильньм" многогранником, которые входят в схему наших исследований. Отметим, что задача нахождения интерполяционных пространств между анизотропными пространствами разной анизотропии интересовала Х. Трибеля (см. [2]). В работе [3] мы доказали соответствующие интерполящионные теоремы в предположении компактного вложения одного из интерполируемых пространств в другое. В терминах порождающих функций $\nu(\xi)$ это условие означает, что $\nu_{0}(\xi) \nu_{1}^{-1}(\xi) \rightarrow+\infty$ при $|\xi| \rightarrow+\infty$.

В настояшей работе нам в несколько шагов удается освободиться от этого ограничения. Сначала исследуется пара пространств, вложенных одно в другое, с порождающими функциями, отношение которых не обязано стремиться к бесконечности. Такие пары мы назьваем соприкасающимися (в терминах многогранников это означает, что соответствующие многогранники имеют общие точки на некоординатных гранях).

Далее, мы доказываем, что при $\theta=1 / 2$ интерполяционные пространства, полученные методом “вещественной” интерполящии для пар $\left\{H\left(\nu_{0}\right), H\left(\nu_{1}\right)\right\}$ и $\{\Sigma(H), \Delta(H)\}$ совпадают (символы $\Sigma(H)$ и $\Delta(H)$ означают сумму и пересечение банаховых пространств $H\left(\nu_{0}\right)$ и $\left.H\left(\nu_{1}\right)\right)$. Поскольку пространства $\Sigma(H)$ и $\Delta(H)$ соприкасаются (см. теорему 2), мы получаем возможность описания интерполяционных пространств для пар пространств типа Соболева-Лиувилля и Никольского-Бесова. 
Доказанные интерполящионные формулы находят свое применение в теории вложений (разных метрик и различных измерений), в теории граничных задач для общих регулярньх дифференциальных операторов и в ряде других вопросов теории функциональных пространств.

1. Интерполяция соприкасающихся пространств. Обозначим через $G_{0}^{+}$множество положительных функций $\mu(\xi)$, бесконечно дифференцируемых вне начала координат и таких, что

$$
\begin{gathered}
\lim _{|\xi| \rightarrow 0} \mu(\xi)=0, \quad \lim _{|\xi| \rightarrow+\infty} \mu(\xi)=+\infty, \quad\left|\xi^{\alpha} D^{\alpha} \mu(\xi)\right| \leqslant c \mu(\xi), \\
\alpha=\left(\alpha_{1}, \ldots, \alpha_{n}\right), \quad \alpha_{i}=0,1, \quad i=1, \ldots, n, \quad \xi \in \mathbb{R}_{n}, \quad \prod_{i=1}^{n} \xi_{i} \neq 0, \quad c>0 .
\end{gathered}
$$

Функции, удовлетворяющие оценке (1), рассматривались, например в [4].

ОПРЕДЕЛЕНИЕ 1 . Пусть $1<p<\infty,-\infty<s<\infty, \mu \in G_{0}^{+}$. Положим

$$
H_{p}^{s}(\nu) \equiv H_{p}^{s}\left(\nu ; \mathbb{R}_{n}\right)=\left\{f \in S^{\prime}:\|f\|_{H}=\left\|F^{-1}\left\{\nu^{s} F f\right\}\right\|_{L_{p}\left(\mathbb{R}_{n}\right)}<\infty\right\},
$$

где $\nu(\xi)=\left(1+\mu^{2}(\xi)\right)^{1 / 2}$.

ОПРЕДЕЛЕНИЕ 2 . Пусть $\mu \in G_{0}^{+}$. Через $\Phi(\mu) \equiv \Phi\left(\mu ; \mathbb{R}_{n}\right)$ обозначим множество систем функций $\left\{\varphi_{k}(\mu ; x)\right\}_{k=0}^{\infty}$, обладаюших следуюшими свойствами:

a) $\varphi_{k} \in S\left(\mathbb{R}_{n}\right),\left(F \varphi_{k}\right)(\xi) \geqslant 0, k=0,1, \ldots$;

б) $\operatorname{supp} F \varphi_{k} \subset\left\{\xi \in \mathbb{R}_{n}: 2^{k-1} \leqslant \mu(\xi) \leqslant 2^{k+1}\right\}, k=1,2, \ldots, \operatorname{supp} F \varphi_{0} \subset\left\{\xi \in \mathbb{R}_{n}\right.$ : $\mu(\xi) \leqslant 2\}$

в) $\sum_{k=0}^{\infty}\left(F \varphi_{k}\right)(\xi)=1, \xi \in \mathbb{R}_{n}$;

г) $\left\|F \varphi_{k}\right\|_{M_{p}} \leqslant c, k=1,2, \ldots, c>0$.

Пример системы из $\Phi(\mu)$ приведен в [5].

ОПрЕДЕЛЕНИЕ 3 . Пусть $-\infty<s<\infty, 1<p<\infty, 1 \leqslant q<\infty, \mu \in G_{0}^{+}$, $\left\{\varphi_{k}\right\}_{k=0}^{\infty} \in \Phi(\mu)$. Положим

$$
B_{p, q}^{s}(\mu)=\left\{f \in S^{\prime}:\|f\|_{B}=\left(\sum_{k=0}^{\infty} 2^{k s q}\left\|f * \varphi_{k}\right\|_{L_{p}\left(\mathbb{R}_{n}\right)}^{q}\right)^{1 / q}<\infty\right\} .
$$

В определениях 1-3 $S$ - класс Шварца, $F$ - оператор преобразования Фурье, $M_{p}-$ пространство мультипликаторов Фурье типа $(p, p)($ см. [1]).

Для функций $\mu(\xi)$, не бесконечно дифференцируемых на координатных осях, $H$ - и $B$-классы определяются как пополнение $S$ в определенных выше нормах.

TЕорема 1. Пусть $0<\theta<1,1 \leqslant q_{0}, q_{1}, r<\infty, 1<p<\infty, \mu_{0}, \mu_{1} \in G_{0}^{+} u$ выполняется неравенство

$$
1+\mu_{1}(\xi) \leqslant c\left(1+\mu_{0}(\xi)\right), \quad \xi \in \mathbb{R}_{n}, \quad c>0
$$

Toгдa

a) $\left(B_{p, q_{0}}^{1}\left(\mu_{0}\right), B_{p, q_{1}}^{1}\left(\mu_{1}\right)\right)_{\theta, r}=B_{p, r}^{1}\left(\mu_{0}^{1-\theta} \cdot \mu_{1}^{\theta}\right)$;

б) $\left(H_{p}^{1}\left(\nu_{0}\right), H_{p}^{1}\left(\nu_{1}\right)\right)_{\theta, r}=B_{p, r}^{1}\left(\mu_{0}^{1-\theta} \cdot \mu_{1}^{\theta}\right)$. 
ДокАЗАтЕльство. Докажем а); б) доказывается аналогично. Воспользуемся теоремой вложения разных метрик для $B$-пространств (см. [6, теорема 2]). Имеем

$$
B_{p, q_{0}}^{1+\varepsilon}\left(\mu_{0}\right) \subset B_{p, q_{0}}^{1}\left(\mu_{0}\right), \quad B_{p, q_{1}}^{1}\left(\mu_{1}\right) \subset B_{p, q_{1}}^{1-\varepsilon}\left(\mu_{1}\right), \quad \varepsilon>0,
$$

причем константы, фигурирующие в этих вложениях, не зависят от $\varepsilon$. Тогда (интерполящионное свойство)

$$
\left(B_{p, q_{0}}^{1+\varepsilon}\left(\mu_{0}\right), B_{p, q_{1}}^{1}\left(\mu_{1}\right)\right)_{\theta, r} \subset\left(B_{p, q_{0}}^{1}\left(\mu_{0}\right), B_{p, q_{1}}^{1}\left(\mu_{1}\right)\right)_{\theta, r} \subset\left(B_{p, q_{0}}^{1}\left(\mu_{0}\right), B_{p, q_{1}}^{1-\varepsilon}\left(\mu_{1}\right)\right)_{\theta, r}
$$

Из оценки (2) видно, что к интерполяционым пространствам слева и справа в (3) применима теорема 7 из [3]. Тогда (3) принимает вид

$$
B_{p, r}^{1}\left(\mu_{0}^{(1+\varepsilon)(1-\theta)} \cdot \mu_{1}^{\theta}\right) \subset\left(B_{p, q_{0}}^{1}\left(\mu_{0}\right), B_{p, q_{1}}^{1}\left(\mu_{1}\right)\right)_{\theta, r} \subset B_{p, r}^{1}\left(\mu_{0}^{1-\theta} \cdot \mu_{1}^{(1-\varepsilon) \theta}\right) .
$$

Для краткости записи перепишем (4) в виде $X \subset Y \subset Z$ и положим, как и в определении 1 ,

$$
\nu_{i}(\xi)=\left(1+\mu_{i}^{2}(\xi)\right)^{1 / 2}, \quad i=0,1
$$

Используя теоремы 4,6 из [3] и правое вложение в (4), получаем для $f \in S$ (из соображений плотности можно ограничиться функциями из $S$ )

$$
\begin{aligned}
\|f\|_{Z}^{r} & \sim \sum_{k=0}^{\infty}\left\|F^{-1}\left\{\nu_{0}^{1-\theta} \cdot \nu_{1}^{(1-\varepsilon) \theta} F \varphi_{k}\left(\mu_{1}\right) F f\right\}\right\|_{L_{p}}^{r} \\
& \sim \sum_{k=0}^{\infty} 2^{-\varepsilon \theta r k}\left\|F^{-1}\left\{\nu_{0}^{1-\theta} \cdot \nu_{1}^{\theta} F \varphi_{k}\left(\mu_{1}\right) F f\right\}\right\|_{L_{p}}^{r} \leqslant c^{\prime}\|f\|_{Y}^{r},
\end{aligned}
$$

где $c^{\prime}>0$ не зависит от $\varepsilon$ и знак ' ' означает двустороннюю оценку. Аналогично, из левого вложения в (4) имеем

$$
\|f\|_{Y}^{r} \leqslant c^{\prime \prime}\|f\|_{X}^{r} \sim \sum_{k=0}^{\infty} 2^{\varepsilon(1-\theta) r k}\left\|F^{-1}\left\{\nu_{0}^{1-\theta} \cdot \nu_{1}^{\theta} F \varphi_{k}\left(\mu_{0}\right) F f\right\}\right\|_{L_{p}}^{r} .
$$

Если обозначить

$$
I f=F^{-1}\left\{\nu_{0}^{1-\theta} \cdot \nu_{1}^{\theta} F f\right\},
$$

то (6) и (7) можно переписать в виде

$$
c_{1}\|I f\|_{B_{p, r}^{-\varepsilon \theta}\left(\mu_{1}\right)} \leqslant\|f\|_{Y} \leqslant c_{0}\|I f\|_{B_{p, r}^{\varepsilon(1-\theta)}\left(\mu_{0}\right)},
$$

где $c_{0}, c_{1}$ не зависят от $\varepsilon$.

Поскольку $f \in S$, то ряды слева и справа в (8) сходятся равномерно (по $\varepsilon$ ) на отрезке $[0,1]$ (в качестве мажорантного ряда можно взять $\|I f\|_{B_{p, r}^{1}\left(\mu_{0}\right)}$ ). Тогда при $\varepsilon \rightarrow 0$, учитывая теоремы 4,6 из [3], получаем

$$
\|f\|_{Y} \sim\|I f\|_{B_{p, r}^{0}} \sim\|f\|_{B_{p, r}^{1}\left(\mu_{0}^{1-\theta} \cdot \mu_{1}^{\theta}\right)^{.}}
$$

Теорема доказана. 
2. Интерполяция суммы и пересечения. Обозначим через $\Sigma(H), \Delta(H)$ (соответственно $\Sigma(B), \Delta(B))$ сумму и пересечение пары пространств $\left\{H_{p}^{1}\left(\nu_{0}\right), H_{p}^{1}\left(\nu_{1}\right)\right\}$ (соответственно $\left.\left\{B_{p, q}^{1}\left(\mu_{0}\right), B_{p, q}^{1}\left(\mu_{1}\right)\right\}\right)$ (см., например, [7]).

Tеорема 2. Пусть $1<p<\infty, \mu_{0}, \mu_{1} \in G_{0}^{+}$. Тогда

a) $\Delta(H)=H_{p}^{1}\left(\nu_{0}+\nu_{1}\right)$;

б) $\Sigma(H)=H_{p}^{1}\left(\frac{\nu_{0} \cdot \nu_{1}}{\nu_{0}+\nu_{1}}\right)$.

Утверждения а), б) верны и для В-пространств.

ДокАЗАТЕЛЬСтво. Утверждение а) непосредственно следует из теоремы П. И. Лизоркина о мультипликаторах Фурье (см. [8]).

Положим $H_{i}=H_{p}^{1}\left(\nu_{i}\right), i=0,1, H=H_{p}^{1}\left(\nu_{0} \cdot \nu_{1} /\left(\nu_{0}+\nu_{1}\right)\right)$. Из теоремы о мультипликаторах Фурье (см. [1]) имеем для $f=f_{0}+f_{1}, f_{i} \in H_{i}$,

$$
\|f\|_{H} \leqslant\left\|f_{0}\right\|_{H}+\left\|f_{1}\right\|_{H} \leqslant c\left(\left\|f_{0}\right\|_{H_{0}}+\left\|f_{1}\right\|_{H_{1}}\right), \quad i=0,1 .
$$

Взяв нижнюю грань по всем разложениям, получаем

$$
\|f\|_{H} \leqslant c\|f\|_{\Sigma(H)}
$$

Докажем обратную оценку. Пусть $f \in H$. Положим (см. [5])

$$
f_{0}=F^{-1}\left\{\frac{\nu_{1} F f}{\nu_{0}+\nu_{1}}\right\}, \quad f_{1}=F^{-1}\left\{\frac{\nu_{0} F f}{\nu_{0}+\nu_{1}}\right\} .
$$

Ясно, что $f_{i} \in H_{i}, i=0,1$, и

$$
\left\|f_{i}\right\|_{H_{i}}=\|f\|_{H}, \quad f=f_{0}+f_{1} .
$$

Тогда

$$
\|f\|_{\Sigma(H)} \leqslant\left\|f_{0}\right\|_{H_{0}}+\left\|f_{1}\right\|_{H_{1}}=2\|f\|_{H} .
$$

Аналогично доказываются утверждения и для $B$-пространств. Теорема доказана.

Из доказательства видно, что соответствующие утверждения верны и при отрицательных верхних индексах.

Teopema 3. Пусть $1<p, q<\infty, \mu_{0}, \mu_{1} \in G_{0}^{+}$. Тогда

$$
(\Sigma(H), \Delta(H))_{1 / 2, q}=\left(H_{0}, H_{1}\right)_{1 / 2, q} .
$$

Утверждение верно и при замене $H$-пространств на В-пространства с фиксированными нижними индексами. 
ДокАЗАТЕЛЬСтво. Поскольку $H$-пространства рефлексивны и $S$ плотно в $H_{p}^{s}(\nu)$ при $-\infty<s<\infty, \mu \in G_{0}^{+}, 1<p<\infty$, то, применяя теорему 2.7.1 из [7], при $0<\theta<1$ получаем

$$
\left(H_{0}, H_{1}\right)_{\theta, q}+\left(H_{1}, H_{0}\right)_{\theta, q}=\left[\left(H_{0}, H_{1}\right)_{\theta, q}^{*} \cap\left(H_{1}, H_{0}\right)_{\theta, q}^{*}\right]^{*} .
$$

Знаком ' $*$ ' помечено сопряженное пространство.

Интерполяционная пара $\left\{H_{0}, H_{1}\right\}$ квазилинеаризуема (см. [9, определение 1.8.4]). Действительно, соответствующие операторы $\left\{V_{0}(t), V_{1}(t)\right\}, 0<t<\infty$, можно определить соотношениями

$$
V_{0}(t) a=F^{-1}\left\{\frac{t \nu_{1} F a}{\nu_{0}+t \nu_{1}}\right\}, \quad V_{1}(t) a=F^{-1}\left\{\frac{\nu_{0} F a}{\nu_{0}+t \nu_{1}}\right\}, \quad a \in \Sigma(H) .
$$

Аналогично тому, как это делалось в теореме 2 , используя теорему Лизоркина о мультипликаторах Фурье (см. оценку (1)), убеждаемся, что операторы из (10) удовлетворяют всем требованиям определения 1.8.4 из [9]. Тогда на основании теоремы 2 можно утверждать, что пара $\{\Sigma(H), \Delta(H)\}$ тоже квазилинеаризуема (соответствующие операторы можно выписать аналогично (10)). Теперь ясно, что выполнены условия теоремы Петре (см. [9, теорема 1.12.1]). Тогда

$$
(\Sigma(H), \Delta(H))_{\theta, q}=\left(\Sigma(H), H_{0}\right)_{\theta, q} \cap\left(\Sigma(H), H_{1}\right)_{\theta, q} .
$$

Все предыдущие рассуждения верны и для пары сопряженных пространств $\left\{H_{0}^{*}, H_{1}^{*}\right\}$ (учитывая, что $\left.\left(H_{p}^{1}(\nu)\right)^{*}=H_{p^{\prime}}^{-1}(\nu)\right)$.

Из (11) при $0<\theta<1,1<q<\infty$ имеем

$$
\begin{gathered}
(\Sigma(H), \Delta(H))_{\theta, q} \supset\left(H_{1}, H_{0}\right)_{\theta, q} \cap\left(H_{0}, H_{1}\right)_{\theta, q}, \\
\left(\Sigma\left(H^{*}\right), \Delta\left(H^{*}\right)\right)_{\theta, q} \supset\left(H_{1}^{*}, H_{0}^{*}\right)_{\theta, q} \cap\left(H_{0}^{*}, H_{1}^{*}\right)_{\theta, q} .
\end{gathered}
$$

Здесь

$$
\Sigma\left(H^{*}\right)=H_{0}^{*}+H_{1}^{*}, \quad \Delta\left(H^{*}\right)=H_{0}^{*} \cap H_{1}^{*} .
$$

Далее, из (9), (13) и теорем 2.7.1 и 3.7.1 из [7] имеем при $1 / q+1 / q^{\prime}=1$

$$
\begin{aligned}
\left(H_{0}, H_{1}\right)_{\theta, q}+\left(H_{1}, H_{0}\right)_{\theta, q} & =\left[\left(H_{0}^{*}, H_{1}^{*}\right)_{\theta, q^{\prime}} \cap\left(H_{1}^{*}, H_{0}^{*}\right)_{\theta, q^{\prime}}\right]^{*} \supset\left(\Sigma\left(H^{*}\right), \Delta\left(H^{*}\right)\right)_{\theta, q^{\prime}}^{*} \\
& =(\Delta(H), \Sigma(H))_{\theta, q^{*}}
\end{aligned}
$$

Формулы (12) и (14) при $\theta=1 / 2$ доказывают утверждение теоремы.

3. Интерполяция пространств $H_{p}^{1}\left(\nu ; \mathbb{R}_{n}\right)$ и $B_{p, q}^{1}\left(\mu ; \mathbb{R}_{n}\right)$. Предыдущие рассмотрения позволяют освободиться от ограничения (2) в теореме 1.

Tеорема 4. Пусть $0<\theta<1,1<p, p_{0}, p_{1}, q_{0}, q_{1}, r<\infty, \mu_{0}, \mu_{1} \in G_{0}^{+}, 1 / p^{*}=$ $(1-\theta) / p_{0}+\theta / p_{1}, 1 / q^{*}=(1-\theta) / q_{0}+\theta / q_{1}$, функиии $\nu_{i}(\xi)$ определены в (5). Тогда
a) $\left[B_{p_{0}, q_{0}}^{1}\left(\mu_{0}\right), B_{p_{1}, q_{1}}^{1}\left(\mu_{1}\right)\right]_{\theta}=B_{p^{*}, q^{*}}^{1}\left(\mu_{0}^{1-\theta} \cdot \mu_{1}^{\theta}\right)$;
б) $\left(B_{p, q_{0}}^{1}\left(\mu_{0}\right), B_{p, q_{1}}^{1}\left(\mu_{1}\right)\right)_{\theta, r}=B_{p, r}^{1}\left(\mu_{0}^{1-\theta} \cdot \mu_{1}^{\theta}\right)$;
в) $\left(H_{p}^{1}\left(\nu_{0}\right), H_{p}^{1}\left(\nu_{1}\right)\right)_{\theta, r}=B_{p, r}^{1}\left(\mu_{0}^{1-\theta} \cdot \mu_{1}^{\theta}\right)$;
г) $\left(B_{p, q_{0}}^{1}\left(\mu_{0}\right), H_{p}^{1}\left(\mu_{1}\right)\right)_{\theta, r}=B_{p, r}^{1}\left(\mu_{0}^{1-\theta} \cdot \mu_{1}^{\theta}\right)$. 
ДокАЗАТЕльСтво. Пусть $\left\{\varphi_{k}(\mu)\right\}_{k=0}^{\infty} \in \Phi(\mu), \mu_{0}^{1-\theta} \cdot \mu_{1}^{\theta}=\mu, I_{\nu_{i}} f=F^{-1}\left\{\nu_{i} F f\right\}$, $i=0,1$. Используя теоремы 4,6 из [3], имеем

$$
\|f\|_{B_{p_{i}, q_{i}}^{1}\left(\mu_{i}\right)} \sim\left\|I_{\nu_{i}} f\right\|_{B_{p_{i}, q_{i}}^{0}\left(\mu_{i}\right)} \sim\left\|I_{\nu_{i}} f\right\|_{B_{p_{i}, q_{i}}^{0}(\mu)}, \quad i=0,1
$$

Если для банахова пространства $A$ положить (см. [7])

$$
\left\|a_{k}\right\|_{\ell_{q}^{s}(A)}=\left(\sum_{k=0}^{\infty} 2^{k s q}\left\|a_{k}\right\|_{A}^{q}\right)^{1 / q}
$$

то соотношения (15) можно переписать в виде

$$
\|f\|_{B_{p_{i}, q_{i}}^{1}\left(\mu_{i}\right)} \sim\left\|I_{\nu_{i}}\left(f * \varphi_{k}(\mu)\right)\right\|_{\ell_{q_{i}}^{0}\left(L_{p_{i}}\right)}=\left\|f * \varphi_{k}(\mu)\right\|_{\ell_{q_{i}}^{0}\left(H_{p_{i}}^{1}\left(\nu_{i}\right)\right)}, \quad i=0,1
$$

Интерполяцией соотношений (16), используя теорему 5.6.3 из [7], теоремы 4.2.2, 5.1.2 из [4] и теорему 6 из [3], приходим к утверждению а) теоремы 4. Докажем б). Пусть сначала $q_{0}=q_{1}=q$. Тогда теоремы $1-3$ доказывают утверждение б) при $\theta=1 / 2$. Пусть $0<\theta, \theta_{0}, \theta_{1}<1, \theta=\left(1-\theta_{0}\right) / 2+\theta_{1} / 2$. Тогда на основании доказанного утверждения а) и теоремы 4.7.2 из [7] имеем

$$
\left(B_{p, q}^{1}\left(\mu_{0}\right), B_{p, q}^{1}\left(\mu_{1}\right)\right)_{\theta, r}=\left(B_{p, q}^{1}\left(\mu_{0}^{1-\theta_{0}} \cdot \mu_{1}^{\theta_{0}}\right), B_{p, q}^{1}\left(\mu_{0}^{1-\theta_{1}} \cdot \mu_{1}^{\theta_{1}}\right)\right)_{1 / 2, r} .
$$

Утверждение б) при $q_{0}=q_{1}$, теперь следует из теорем $1-3$. Пусть $q_{0} \neq q_{1}$. Тогда для $q=\max \left(q_{0}, q_{1}\right)$ имеем из доказанного

$$
\left(B_{p, q_{0}}^{1}\left(\mu_{0}\right), B_{p, q_{1}}^{1}\left(\mu_{1}\right)\right)_{\theta, r} \subset\left(B_{p, q}^{1}\left(\mu_{0}\right), B_{p, q}^{1}\left(\mu_{1}\right)\right)_{\theta, r}=B_{p, r}^{1}(\mu)
$$

Аналогично доказьвается обратное вложение, используя $q=\min \left(q_{0}, q_{1}\right)$. Утверждение б) доказано.

Поскольку

$$
\left(H_{p}^{1}\left(\nu_{0}\right), H_{p}^{1}\left(\nu_{1}\right)\right)_{\theta_{i}, q_{i}} \subset\left(B_{p, \max (p, 2)}^{1}\left(\mu_{0}\right), B_{p, \max (p, 2)}^{1}\left(\mu_{1}\right)\right)_{\theta_{i}, q_{i}}, \quad i=0,1
$$

то интерполяцией $\left(\theta=(1-\eta) \theta_{0}+\eta \theta_{1}\right)$ из теоремы реитерации 3.5 .3 из [7] и утверждения б) получаем

$$
\left(H_{p}^{1}\left(\nu_{0}\right), H_{p}^{1}\left(\nu_{1}\right)\right)_{\theta, r} \subset B_{p, r}^{1}(\mu) .
$$

Аналогично доказывается обратное вложение. Утверждение в) доказано. Утверждение г) доказывается аналогично. Теорема доказана. 


\section{СПИСОК ЦИТИРОВАННОЙ ЛИТЕРАТУРЫ}

[1] Никольский С. М. Приближение функций многих переменных и теоремы вложения. М.: Наука, 1977.

[2] Трибель Х. Теория функциональных пространств. М.: Мир, 1986.

[3] Багдасарян А. Г. Интерполяция некоторых функциональных пространств разной анизотропии // Изв. АН Армении. 1992. Т. 27. № 4. С. 49-58.

[4] Triebel H. General function spaces III (spaces $B_{p, q}^{s(x)}, F_{p, q}^{s(x)}, 1<p<\infty$ : basic properties) // Anal. Math. 1977. V. 3. P. 221-249.

[5] Багдасарян А. Г. Об интерполяции и следах функций из некоторых анизотропных функциональных пространств // Изв. АН АрмССР. 1988. Т. 23. № 4. С. 353-365.

[6] Багдасарян А.Г. Теоремы вложения для некоторых обобщенных функциональных пространств // Изв. АН Армении. 1994. Т. 29. № 3. С. 1-10.

[7] Берг $\breve{И} .$, Лёфстрём Й. Интерполяционные пространства. Введение. М.: Мир, 1980.

[8] Лизоркин П.И. $\left(L_{p}, L_{q}\right)$-мультипликаторы интегралов Фурье // Докл. АН СССР. 1963. T. 152. № 4. C. 808-811.

[9] Трибель Х. Теория интерполяции, функциональные пространства, дифференциальные операторы. М.: Мир, 1980.

Ереванский государственный университет

Поступило

04.07 .96 